\title{
Certain subphenotypes of aspirin-exacerbated respiratory disease distinguished by latent class analysis
}

\author{
Grazyna Bochenek, MD, PhD, Joanna Kuschill-Dziurda, MD, ${ }^{a}$ Krystyna Szafraniec, PhD, ${ }^{\text {b }}$ Hanna Plutecka, PhD, \\ Andrzej Szczeklik, MD, PhD, ${ }^{{ } \dagger}$ and Ewa Nizankowska-Mogilnicka, MD, PhD ${ }^{\mathrm{a}}$ Krakow, Poland
}

Background: Aspirin-exacerbated respiratory disease (AERD) is recognized as a distinct asthma phenotype. It usually has a severe course accompanied by chronic hyperplastic eosinophilic sinusitis with nasal polyps, blood eosinophilia, and increased concentrations of urinary leukotriene $\mathbf{E}_{4}\left(\mathrm{LTE}_{4}\right)$. More insightful analysis of individual patients shows this group to be nonhomogeneous.

Objective: We sought to identify any likely subphenotypes in a cohort of patients with AERD through the application of latent class analysis (LCA).

Methods: Clinical data from 201 patients with AERD (134 women) were collected from questionnaires. Standard spirometry, atopy traits, blood eosinophilia, and urinary $\mathrm{LTE}_{4}$ concentrations were evaluated. LCA was applied to identify possible AERD subphenotypes.

Results: Four classes (subphenotypes) within the AERD phenotype were identified as follows: class 1 , asthma with a moderate course, intensive upper airway symptoms, and blood eosinophilia (18.9\% of patients); class 2 , asthma with a mild course, relatively well controlled, and with low health care use (34.8\% of patients); class 3 , asthma with a severe course, poorly controlled, and with severe exacerbations and airway obstruction ( $41.3 \%$ of patients); and class 4 , poorly controlled asthma with frequent and severe exacerbations in female subjects (5.0\% of patients). Atopic status did not affect class membership. Patients with particularly intensive upper airway symptoms had the highest levels of blood eosinophilia and the highest concentrations of urinary $\mathrm{LTE}_{4}$.

Conclusions: LCA revealed unique AERD subphenotypes, thus corroborating the heterogeneity of this population. Such discrimination might facilitate more individualized treatment in difficult-to-treat patients. ( $\mathrm{J}$ Allergy Clin Immunol 2014;133:98-103.)

Key words: Aspirin, aspirin-exacerbated respiratory disease, asthma phenotype, latent class analysis, nonsteroidal antiinflammatory drugs

\footnotetext{
From ${ }^{a}$ the Department of Internal Medicine and ${ }^{b}$ the Department of Epidemiology and Population Studies, Institute of Public Health, Jagiellonian University Medical College.

$\dagger$ Deceased.

Supported by a grant from the Polish Ministry of Science and Higher Education and Jagiellonian University (K/ZDS/001022).

Disclosure of potential conflict of interest: The authors have been supported by a grant from the Polish Ministry of Science and Higher Education (no. K/ZDS/001022).

Received for publication February 23, 2013; revised June 15, 2013; accepted for publication July 9, 2013

Available online August 27, 2013

Corresponding author: Grazyna Bochenek, MD, PhD, Department of Internal Medicine, Jagiellonian University Medical College, Skawinska 8, 31-066 Krakow, Poland. E-mail: mmbochen@cyf-kr.edu.pl.

0091-6749/\$36.00

(C) 2013 American Academy of Allergy, Asthma \& Immunology

http://dx.doi.org/10.1016/j.jaci.2013.07.004
}

Abbreviations used

AERD: Aspirin-exacerbated respiratory disease

cysLT: Cysteinyl leukotriene

ED: Emergency department

ICU: Intensive care unit

LCA: Latent class analysis

LTE $_{4}$ : Leukotriene $\mathrm{E}_{4}$

NAEPP EPR3: National Asthma Education and Prevention Program Expert Panel Report 3

NSAID: Nonsteroidal anti-inflammatory drug

TENOR: The Epidemiology and Natural History of Asthma: Outcomes and Treatment Regimens

Aspirin-exacerbated respiratory disease (AERD) is a distinct clinical syndrome characterized by chronic eosinophilic inflammation of the upper and lower airways with symptoms that are exacerbated by aspirin and other nonsteroidal anti-inflammatory drugs (NSAIDs) ${ }^{1-3}$ It is generally recognized as severe asthma with a predilection for female subjects that develops in line with a characteristic sequence of symptoms. ${ }^{2}$ The majority of patients have chronic hyperplastic eosinophilic sinusitis with nasal polyps characterized by rapid regrowth, resulting in multiple sinus surgeries. ${ }^{4}$

Atopy is rather frequent in this population. ${ }^{4,5}$ Most patients with AERD synthesize excessive amounts of cysteinyl leukotrienes (cysLTs) in a stable condition, which is reflected by increased urinary leukotriene $\mathrm{E}_{4}\left(\mathrm{LTE}_{4}\right)$ concentrations when compared with those seen in asthmatic patients who tolerate aspirin well. ${ }^{6}$

In view of this relatively distinct clinical and pathophysiologic presentation, AERD is regarded as one of the specific asthma phenotypes. ${ }^{7}$ When individual cases are more insightfully analyzed, however, this group no longer appears to be so homogenous, differing in terms of specific clinical and laboratory parameters.

Clinical studies carried out to date on numerous groups of patients with AERD have analyzed the prevalence or mean values of several parameters and subsequently proposed the average clinical picture of this asthma phenotype. ${ }^{2,4}$ Another approach compared a large group of patients with AERD with patients with aspirin-tolerant asthma in terms of several differentiating clinical variables. ${ }^{8}$

The present study aimed to identify and describe the likely subphenotypes within a cohort of patients representing the AERD phenotype by applying advanced statistical modeling methods. As opposed to the previously referenced variable-oriented studies, the present study was focused on patients. Hence we applied latent class analysis (LCA) to group together patients with AERD who were similar to each other in terms of the selected clinical variables.

With regard to respiratory diseases, this statistical approach had been applied in large populations, which effectively helped to 
identify several wheezing and asthma phenotypes. ${ }^{9-11}$ Another methodology (ie, a hierarchic cluster analysis) facilitated the identification of distinct clinical phenotypes in asthmatic adults and children. ${ }^{12-14}$

\section{METHODS}

\section{Subjects studied}

The participants were recruited from consecutive patients given a diagnosis of AERD at the Department of Internal Medicine, Jagiellonian University Medical College, Krakow, Poland. The recruitment phase spanned from June 2008 to September 2010. In total, 201 patients were enrolled. They remained without any asthma exacerbations in the 4 weeks preceding the study and received asthma medications as currently prescribed by their physicians. All patients signed informed consent forms approved by the Jagiellonian University Ethics Review Committee.

The AERD diagnosis was made before the study. It was based on a typical history confirmed by a positive oral or inhaled aspirin challenge result. ${ }^{15}$ In 42 patients with severe steroid-dependent asthma, the diagnosis had to be based exclusively on the unequivocal clinical picture and a history of asthma attacks after ingestion of NSAIDs. Because of both low $\mathrm{FEV}_{1}$ and the nonfeasibility of reducing the dosage of oral corticosteroids, these patients never qualified for the aspirin challenge. ${ }^{15}$

\section{Data collection}

Patients' data were collected from a specifically structured questionnaire. Participants underwent a demographic and detailed medical history interview. The current level of asthma severity was based on the National Asthma Education and Prevention Program Expert Panel Report 3 (NAEPP EPR3). ${ }^{16}$ Asthma control was assessed by using the Asthma Control Test. Standard spirometry and skin prick tests were performed. Blood eosinophilia, total IgE levels, and baseline urinary $\mathrm{LTE}_{4}$ concentrations were measured (see the Methods section in this article' Online Repository at www.jacionline.org).

\section{Analytic strategy and selection of variables}

Latent class models were fitted to the clinical and laboratory variables assessed in the study. ${ }^{17}$ This statistical modeling technique was used to estimate the number of classes of underlying categorical latent variables with a finite number of mutually exclusive levels, which simultaneously considered the relationships between the respective numbers of variables under consideration. Two types of parameters were estimated: the prevalence of each latent class (ie, a priori probability that a selected subject was in each class) and the conditional probabilities describing the distribution of the responses to each question within each class. Identification of the optimal model was done in a stepwise manner (see the Methods section and Table E1 in this article' Online Repository at www.jacionline.org). The best-fitting 4-class model was finally determined. Each subject was allocated to a single latent class based on the maximum-probability assignment rule (ie, to the class with the highest a posteriori probability of membership; see the Methods section and Table E2 in this article' Online Repository at www.jacionline.org).

The applied procedure demonstrated that the membership probability was more than 0.8 for $160(79.6 \%)$ patients and more than 0.9 for $124(61.7 \%)$ patients. Only for $10(5.0 \%)$ patients was the highest membership probability less than 0.6, thus indicating its more ambiguous nature.

Because LCA requires categorization and independence of the considered variables, the following variables relevant to the study were analyzed: asthma age of onset; body mass index; current level of asthma control; asthma-related emergency department (ED) visits, hospitalizations, and stays in the intensive care unit (ICU) through the entire time of asthma duration; upper airway symptoms, occurrence of nasal polyps, and history of polypectomies as indicators of chronic rhinosinusitis; $\mathrm{FEV}_{1}$ percent predicted; $\Delta \mathrm{FEV} \mathrm{F}_{1}$ after bronchodilator; skin prick test responses; total IgE levels; and blood eosinophilia (detailed in the Methods section in this article' Online Repository).
Sex effects and asthma duration were additionally estimated as covariates. The class membership probabilities were estimated separately for male and female patients by using sex as a categorical covariate.

After identifying the 4 final classes, the mean values of the logarithmically transformed urinary $\mathrm{LTE}_{4}$ concentrations were calculated and compared among the classes. In each class treatment of asthma (divided into 4 categories) was assessed, and the proportion of patients using each category of treatment was computed. Finally, the current level of asthma severity was analyzed. In each class the proportion of patients representing each level of asthma severity was computed as well (see the Methods section in this article' Online Repository).

\section{Statistical analysis}

The statistical software SAS version 9.1 (SAS Institute, Cary, NC) was used to calculate the descriptive statistics and to carry out all analyses, including LCA (PROC LCA version 1.2.7). ${ }^{18}$ One-way ANOVA was applied to assess the difference in the logarithmically transformed $\mathrm{LTE}_{4}$ measurements among the 4 classes. The statistical significance was set to an $\alpha$ level of .05 . The odds ratio of being in a given class relative to the fixed reference class for a 1-year increase within asthma duration was estimated by using logistic regression, with the latent class as the dependent variable and asthma duration as the independent variable.

\section{RESULTS}

The clinical characteristics for the entire AERD cohort are presented in Table I. The main characteristics of the respective classes are summarized below (detailed in Table II and Table E3 in this article' Online Repository at www.jacionline.org).

Thirty-eight (18.9\%) patients (mean age, $41.4 \pm 11.5$ years) were allocated to class 1 , which was defined as having "asthma with a moderate course, intensive upper airways symptoms, and blood eosinophilia." This class contained the highest proportion of patients with chronic rhinosinusitis, as determined by upper airway symptoms and the occurrence of nasal polyps. The frequency of asthma-related ED visits was equally distributed within the 3 established intervals. Only half of the patients had a high frequency of hospitalizations, whereas the other half displayed low numbers. Stays in the ICU were very rare. Asthma was partially controlled in half of the patients and uncontrolled in one third, even though $87 \%$ of patients were treated with oral corticosteroids, inhaled corticosteroids, or both (Fig 1). Mild asthma was established in $34 \%$ of patients, moderate in $29 \%$, and severe only in $10.5 \%$ (Fig 2). The highest proportion of patients had increased blood eosinophil counts. This class was also unique in its significantly highest urinary $\mathrm{LTE}_{4}$ concentration (Fig 3).

Seventy $(34.8 \%)$ patients (mean age, $50.1 \pm 13.4$ years) were grouped into class 2 , which was defined as having "asthma with a mild course, relatively well controlled, with low health care use." This group comprised the highest proportion of male patients. The frequency of asthma-related ED visits and hospitalizations was the lowest. This class had the best asthma control, even though $25.7 \%$ of patients remained without any corticosteroid treatment (Fig 1). These patients had milder asthma because $35.7 \%$ had intermittent and $25.7 \%$ had mild disease (Fig 2). The urinary $\mathrm{LTE}_{4}$ concentration was comparable with that in class 3 patients, although significantly lower than in class 1 patients (Fig 3).

Eighty-three $(41.3 \%)$ patients (mean age, $52.7 \pm 10.2$ years) were allocated to class 3 , which was defined as having "asthma with a severe course, poorly controlled, with severe exacerbations and airway obstruction." Female patients outnumbered male 
TABLE I. Characteristics of the entire AERD cohort

\begin{tabular}{|c|c|}
\hline Variables & Parameters \\
\hline No. of participants & 201 \\
\hline Female subjects, no. (\%) & $134(66.6)$ \\
\hline Age $(y)$, mean $\pm S D$ & $49.4 \pm 12.4$ \\
\hline Age at onset of asthma $(y)$, mean \pm SD & $30.4 \pm 11.2$ \\
\hline$\geq 12$ y, no. $(\%)$ & $192(95.5)$ \\
\hline Duration of asthma $(y)$, mean $\pm S D$ & $19.5 \pm 11.1$ \\
\hline $\mathrm{BMI}$, mean $\pm \mathrm{SD}$ & $26.3 \pm 4.5$ \\
\hline$\geq 30 \mathrm{~kg} / \mathrm{m}^{2}$, no. $(\%)$ & $37(18.4)$ \\
\hline \multicolumn{2}{|l|}{ Present level of asthma control (ACT), no. (\%) } \\
\hline 1: Well controlled & $41(20.4)$ \\
\hline 2: Partially controlled & $69(34.2)$ \\
\hline 3: Uncontrolled & $91(45.3)$ \\
\hline ED visits, * median (minimum-maximum) & $2(0-55)$ \\
\hline Hospitalizations, ${ }^{*}$ median (minimum-maximum) & $2(0-36)$ \\
\hline Hospitalizations at ICU, no. (\%) & $47(23.4)$ \\
\hline Upper airway symptoms, no. (\%) & $163(81.1)$ \\
\hline Nasal polyps, whenever, no. (\%) & $162(80.6)$ \\
\hline Polypectomies, whenever, no. (\%) & $133(81.6)$ \\
\hline $\mathrm{FEV}_{1}(\%$ predicted $<80 \%)$, no. $(\%)$ & $98(48.8)$ \\
\hline$\Delta \mathrm{FEV}_{1} \geq 12 \%$ of baseline, no. (\%) & $71(37.4)$ \\
\hline Positive skin prick test responses, no. (\%) & $105(52.2)$ \\
\hline IgE total, median (minimum-maximum) & $87.6(17-2130$ \\
\hline$\geq 100 \mathrm{IU} / \mathrm{mL}$, no. $(\%)$ & $88(43.8)$ \\
\hline Blood eosinophil count, median (minimum-maximum) & $295.0(0-2273)$ \\
\hline$\geq 400 / \mathrm{mL}$, no. $(\%)$ & $82(40.8)$ \\
\hline $\operatorname{logLTE}{ }_{4}(\mathrm{ng} / \mathrm{mg}$ creatinine $)$, mean $\pm \mathrm{SD}$ & $2.91 \pm 0.56$ \\
\hline \multicolumn{2}{|l|}{ Treatment of asthma, no. $(\%)$} \\
\hline Without CS & $29(14.4)$ \\
\hline ICS $\leq 500 \mu \mathrm{g} / \mathrm{d}$ & $46(22.9)$ \\
\hline ICS $>500 \mu \mathrm{g} / \mathrm{d}$ & $66(32.8)$ \\
\hline OCS & $60(29.9)$ \\
\hline \multicolumn{2}{|l|}{ Levels of asthma severity, no. (\%) } \\
\hline Intermittent & 38 (18.9) \\
\hline Mild persistent & $32(15.9)$ \\
\hline Moderate persistent & $70(34.8)$ \\
\hline Severe persistent & $61(30.3)$ \\
\hline
\end{tabular}

$\overline{A C T}$, Asthma Control Test; BMI, body mass index; $C S$, corticosteroids; $I C S$, inhaled corticosteroids; $O C S$, oral corticosteroids.

*Throughout the time of asthma duration.

patients by a factor of 2 . All patients had bronchial obstruction, which was reversible in $61 \%$ of cases. Almost half of the patients had high frequencies of asthma-related ED visits and hospitalizations, whereas $37 \%$ stayed in the ICU. Asthma remained uncontrolled in $71 \%$ of patients, despite the administration of oral corticosteroids in $44 \%$ of patients (Fig 1). The criteria for severe asthma were met in $55 \%$ of patients (Fig 2). Urinary $\mathrm{LTE}_{4}$ concentrations were similar to those in class 2 patients, although significantly lower than in class 1 patients (Fig 3).

Class 4 comprised $10(5.0 \%)$ female patients only (mean age, $46.9 \pm 13.4$ years) and was defined as having "poorly controlled asthma, with frequent and severe exacerbations in female patients." Almost half of the patients had childhood-onset asthma, and $41 \%$ were obese. Almost all patients had high frequencies of asthma-related ED visits and hospitalizations, with the same patterns for the ICU. Asthma remained uncontrolled in $71 \%$ of patients, despite all patients having been started on corticosteroid treatment (Fig 1). The respective patients met the criteria for the different levels of asthma severity, although half had severe disease (Fig 2). Urinary $\mathrm{LTE}_{4}$ concentrations were comparable with those of patients in classes 2 and 3 but lower than in patients
TABLE II. Characteristics of the particular classes and probability of having a patient allocated to one of the 4 classes based on LCA

\begin{tabular}{|c|c|c|c|c|}
\hline & Class 1 & Class 2 & Class 3 & Class 4 \\
\hline Male subjects & 0.13 & 0.47 & 0.40 & 0.00 \\
\hline Female subjects & 0.20 & 0.33 & 0.37 & 0.10 \\
\hline \multicolumn{5}{|l|}{ Age at asthma onset } \\
\hline$\geq 12 \mathrm{y}$ & 0.92 & 1.00 & 1.00 & 0.54 \\
\hline \multicolumn{5}{|l|}{ BMI } \\
\hline$\geq 30 \mathrm{~kg} / \mathrm{m}^{2}$ & 0.05 & 0.17 & 0.23 & 0.41 \\
\hline \multicolumn{5}{|l|}{ Present level of asthma control (ACT) } \\
\hline 1: Well controlled & 0.20 & 0.36 & 0.05 & 0.17 \\
\hline 2: Partially controlled & 0.51 & 0.40 & 0.24 & 0.12 \\
\hline 3: Uncontrolled & 0.29 & 0.24 & 0.71 & 0.71 \\
\hline \multicolumn{5}{|l|}{ ED visits* } \\
\hline First tertile $(<0.63$ per $10 \mathrm{y})$ & 0.33 & 0.48 & 0.25 & 0.01 \\
\hline Second tertile $(0.63-2.57$ per $10 \mathrm{y})$ & 0.36 & 0.38 & 0.31 & 0.15 \\
\hline Third tertile $(>2.57$ per $10 \mathrm{y})$ & 0.31 & 0.14 & 0.44 & 0.84 \\
\hline \multicolumn{5}{|l|}{ Hospitalizations* } \\
\hline First tertile $(<0.5$ per $10 \mathrm{y})$ & 0.51 & 0.46 & 0.16 & 0.09 \\
\hline Second tertile $(0.5-2.11$ per $10 \mathrm{y})$ & 0.01 & 0.46 & 0.40 & 0.24 \\
\hline Third tertile $(>2.11$ per $10 \mathrm{y})$ & 0.48 & 0.08 & 0.44 & 0.67 \\
\hline \multicolumn{5}{|l|}{ Hospitalizations at ICU } \\
\hline Yes & 0.03 & 0.10 & 0.37 & 0.80 \\
\hline \multicolumn{5}{|l|}{ Upper airway symptoms } \\
\hline Yes & 1.00 & 0.70 & 0.85 & 0.68 \\
\hline \multicolumn{5}{|l|}{ Nasal polyps, whenever } \\
\hline Yes & 0.96 & 0.80 & 0.78 & 0.55 \\
\hline \multicolumn{5}{|l|}{ Polypectomies, whenever } \\
\hline Yes & 0.81 & 0.94 & 0.72 & 0.66 \\
\hline \multicolumn{5}{|l|}{$\mathrm{FEV}_{1}(\%$ predicted $)$} \\
\hline$<80 \%$ & 0.20 & 0.16 & 1.00 & 0.21 \\
\hline \multicolumn{5}{|l|}{$\Delta \mathrm{FEV}_{1}$} \\
\hline$\geq 12 \%$ & 0.25 & 0.21 & 0.61 & 0.20 \\
\hline \multicolumn{5}{|l|}{ Positive skin prick test responses } \\
\hline Yes & 0.59 & 0.52 & 0.47 & 0.63 \\
\hline \multicolumn{5}{|l|}{ IgE total } \\
\hline$\geq 100 \mathrm{IU} / \mathrm{mL}$ & 0.67 & 0.16 & 0.60 & 0.45 \\
\hline \multicolumn{5}{|l|}{ Blood eosinophil count } \\
\hline$\geq 400 / \mathrm{mL}$ & 0.63 & 0.31 & 0.40 & 0.41 \\
\hline
\end{tabular}

ACT, Asthma Control Test; BMI, body mass index.

*Throughout the time of asthma duration.

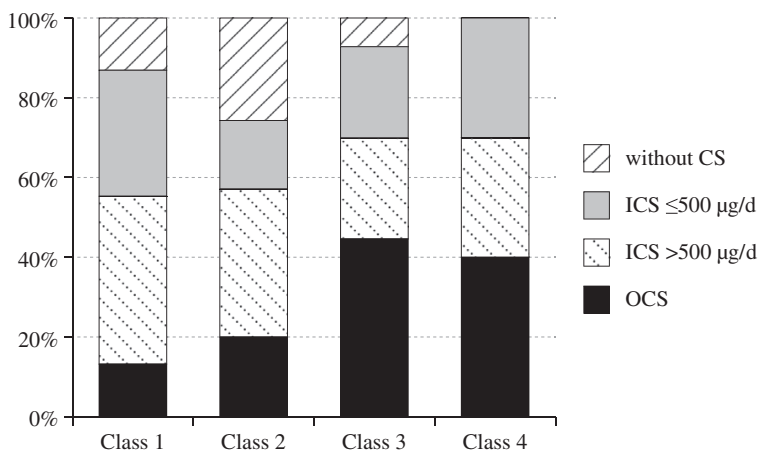

FIG 1. Asthma treatment in the respective classes, as identified by LCA. CS, Corticosteroids; ICS, inhaled corticosteroids; OCS, oral corticosteroids.

in class 1 , although the differences proved statistically insignificant because of the small size of this group (Fig 3).

The odds for the allocation to a particular class were related to asthma duration. Class 3 was assumed as "reference one" 


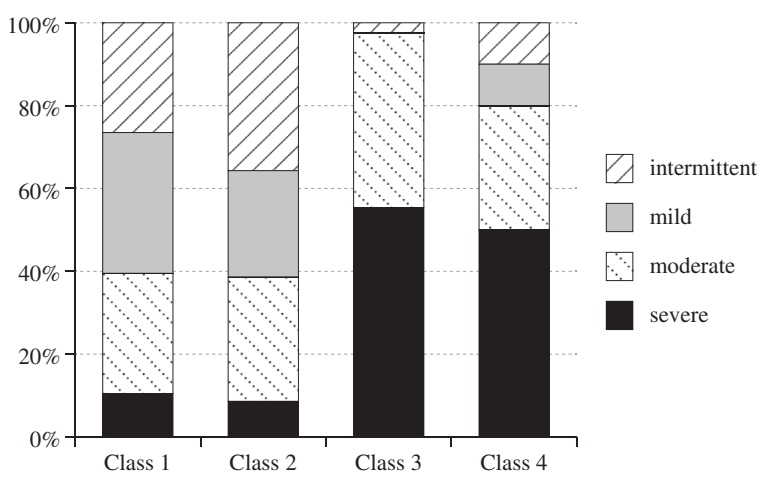

FIG 2. Levels of asthma severity in the respective classes, as identified by LCA.

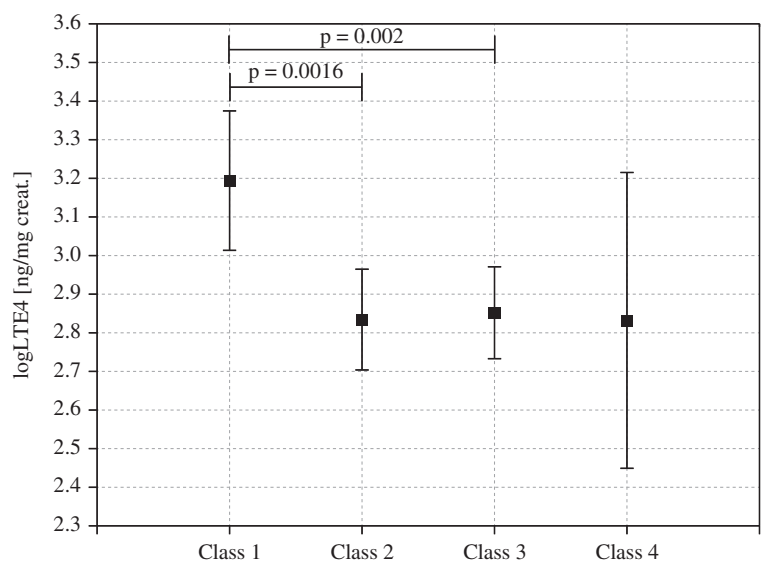

FIG 3. Urinary log $\mathrm{LTE}_{4}$ levels in the 4 classes, as identified by LCA. Values are presented as mean $(95 \% \mathrm{Cls})$.

because it most closely resembled a typical clinical presentation of AERD. The most relevant results were found for male patients allocated to class 2. The effect of asthma duration in female patients was observed in classes 1 and 4, although it lacked the relevance encountered in male patients (see Table E4 in this article' Online Repository at www.jacionline.org).

\section{DISCUSSION}

By applying a novel biostatistical modeling approach, this study was the first to separate 4 subphenotypes (latent classes) within the AERD cohort. We used the LCA method in which patients were grouped together according to the similarities of the specific asthma features.

In the present study, much like in the large cohort studies on AERD, female patients outnumbered male patients. ${ }^{2,4}$ A prior European study demonstrated that female patients had an earlier onset of aspirin hypersensitivity symptoms, more frequent occurrence of nasal polyps, and more severe manifestations of asthma. ${ }^{2}$ Even though the sexes were not compared directly, we noticed that class 4 (female subjects only) was distinguishable by higher use of health care services, which might be indicative of a severe course of asthma. In addition, class 3 , in which female patients outnumbered male patients 2 to 1 , was characterized by a higher frequency of asthma-related ED visits, hospitalizations, and stays in the ICU when compared with classes 1 and 2 . In contrast, class
2 , in which the male/female ratio was in favor of male patients, was characterized by a much less frequent need for health care use.

One of the plausible explanations might be that either female patients are more vulnerable to asthma symptoms or that they are more inclined to seek medical attention. Female asthmatic patients reportedly showed a better appreciation of dyspnea and were more frequent ED visitors than male patients. ${ }^{19,20}$

AERD is usually described as moderate-to-severe asthma requiring continuous systemic corticosteroid therapy in approximately half of all cases, apart from inhaled corticosteroids, to control bronchial and nasal symptoms. ${ }^{2,4}$ In The Epidemiology and Natural History of Asthma: Outcomes and Treatment Regimens (TENOR) study, patients with AERD had more severe manifestations of the disease than did patients with severe aspirintolerant asthma. ${ }^{8}$ In the European Network for Understanding Mechanisms of Severe Asthma (ENFUMOSA) study, aspirin hypersensitivity was associated with more severe cases of asthma. $^{21}$ Kowalski et $\mathrm{al}^{22}$ revealed that hypersensitivity to aspirin and other NSAIDs was a significant risk factor for severe refractory asthma.

We established that patients with the most severe course of asthma were grouped together in classes 3 and 4. For practical purposes, we used the term "severe course of asthma" because it was broader than the term "current level of asthma severity," in a strict sense of the NAEPP EPR3 criteria. ${ }^{16}$ In class 3 this was expressed by the presence of bronchial obstruction in all patients, frequent health care use, lack of asthma control, and the requirement for high doses of inhaled corticosteroids, oral corticosteroids, or both in the majority of patients. Otherwise, class 3 actually included the highest proportion of patients with severe and moderate levels of asthma severity. In many respects this class most closely resembled a typical clinical picture of AERD.

In class 4 the severe course of asthma was reflected by the most frequent health care use and poor asthma control, despite high treatment regimens. As opposed to class 3, the majority of patients in class 4 had normal lung function, which might imply difficult-to-treat severe asthma. ED visits and aspirin-induced asthma attacks have been shown to be independent and crucial risk factors in patients with near-fatal asthma. ${ }^{23}$ Despite its small size, class 4 seemed to inspire some extra interest. It comprised female patients only, almost half of whom were obese and had early-onset asthma. Fukutomi et $\mathrm{al}^{24}$ demonstrated that obesity and aspirin hypersensitivity were the significant risk factors for difficult-to-treat asthma, with this association proved significant only in female patients. Thus far, 2 phenotypes were identified comprising obese women with late-onset severe asthma. ${ }^{12,13}$ Further studies should be pursued with larger groups of obese women with a severe course of AERD to facilitate more reliable conclusions. Regarding the small population size, Wearthall et $\mathrm{al}^{25}$ applied cluster analysis and identified 4 distinct clinical phenotypes of airway diseases in a group of 175 patients. Two clusters comprised only 10 and 11 patients, respectively.

A subgroup of patients with a milder course of AERD was allocated to class 2 . They were characterized by normal lung function, sparse health care use, and overall better asthma control, the last of which was achieved despite less intensive corticosteroid treatment than in class 3 .

The limitation of our study involves the real-life setting (ie, the patients might have been either overtreated or undertreated). The 
fact that some patients remained without any corticosteroid treatment could be attributed to either of the 2 following reasons: either they were not properly treated and hence had severe exacerbations or they had intermittent asthma not requiring a regular corticosteroid treatment. Another limitation could be that the actual reliability of the patients' reported histories of exacerbations, especially in the cases when proper medical documentation was unavailable, were somewhat dubious.

All of the class 3 patients with a more severe course of asthma had bronchial obstruction, which was reversible in $61 \%$ of cases. A vast majority of class 2 patients with a less severe course of asthma had normal lung function. In the TENOR study all patients with AERD had bronchial obstruction, which was constant and refractory to bronchodilators. ${ }^{8}$ This prompted the TENOR investigators to conclude that AERD was characterized by aggressive remodeling of the respiratory tract. The discrepancies between the present study and the TENOR study might arise from the essential differences in the schedules of both studies and the selection of participating patients. Our study was pursued as a real-life, cross-sectional one in which spirometry was performed once. The TENOR study was a 3-year observational study embracing the selected patients with severe or difficult-to-treat asthma in whom spirometry was carried out on multiple occasions. ${ }^{8}$

Nevertheless, we are still unable to ascertain explicitly that airway remodeling applies to all patients with AERD. When the high-resolution computed tomographic technique was applied, no significant differences in air trapping and bronchial wall thickness were observed between aspirin-intolerant and aspirin-tolerant asthmatic patients. ${ }^{26}$ However, such a study should embrace a larger population of patients to secure more conclusive evidence.

Chronic rhinosinusitis with nasal polyps is a typical component of AERD. ${ }^{2,4,27}$ Even though all 4 classes comprised a high proportion of patients with chronic rhinosinusitis, class 1 was unique in this regard. The clinical course of asthma in this class seemed to be more severe than in class 2 but milder than in class 3 when estimated in terms of the demand for health care resources, asthma control, and pulmonary function. Only $20 \%$ of patients had bronchial obstruction. Therefore particularly intensive upper airway disease was not necessarily interrelated with a severe course of asthma. Ponte et $\mathrm{al}^{28}$ demonstrated that moderate-tosevere rhinitis was strongly associated with those parameters indicating greater asthma severity, whereas Williamson et $\mathrm{al}^{29}$ did not find any correlation between the severity of chronic rhinosinusitis and lower airway dysfunction.

Class 1 was also distinguishable by the highest proportion of patients with increased blood eosinophil counts and the highest $\mathrm{LTE}_{4}$ concentrations in urine, which could be directly related to an abundant chronic eosinophilic inflammation of their upper airways. Blood eosinophilia is regarded as a good marker for eosinophilic inflammation of nasal polyps in patients with eosinophilic chronic rhinosinusits. ${ }^{30,31}$ Class 1 comprised also the lowest proportion of patients treated with oral corticosteroids. Very likely, a more intensive treatment with these drugs could have decreased eosinophil counts and alleviated upper airways symptoms as a result.

Most patients with AERD synthesize excessive amounts of cysLTs, for which eosinophils and mast cells are a likely cellular source. ${ }^{6,32}$ Higashi et $\mathrm{al}^{33}$ put forward the working hypothesis that cysLT overproduction was not strictly associated with aspirin intolerance itself but rather with the presence of chronic hyperplastic rhinosinusitis because a significant decrease in urinary $\mathrm{LTE}_{4}$ concentrations was observed after endoscopic surgery for rhinosinusitis. This particular observation remains very much on par with our own results, thereby strongly suggesting the existence of the AERD subphenotype, as already ascertained in class 1 .

The present study revealed that atopic status was not a significant discriminator affecting class membership because approximately half of the patients in each class had positive skin prick test responses. These results did not allow the identification of any particular atopic or nonatopic AERD subphenotypes. The proportion of patients with increased total IgE levels varied considerably between the respective classes and did not correspond with positive skin prick test responses. However, the total IgE level in atopy identification is presently being put into question because of a considerable overlap in levels between atopic and nonatopic populations. ${ }^{34}$

Our LCA revealed discrete clinical subphenotypes within AERD, which is regarded as an asthma phenotype in its own right. The choice of 4 classes seemed to be fully justifiable because it was based on the lowest Akaike information criterion, Bayesian information criterion, and entropy values (see the Results section in this article's Online Repository at www.jacionline.org). Furthermore, high membership probability for the majority of patients indicated that they were allocated in an unambiguous manner. We decided to use 14 variables from the available dataset because they embraced a wide range of clinical traits addressing different aspects of the disease, were independent, and rendered themselves to categorization.

The results might give grounds to some doubt as to whether such a separation of the AERD population into latent classes might actually offer an entirely realistic approach. This is especially the case because each subject can be assigned to various classes with different probabilities, suggesting the feasibility of overlap between the respective classes. In our study such overlap occurred between classes 1 and 2. Furthermore, the distribution of patients between the latent classes might fluctuate in line with the sample size and number and type of variables under consideration. Moreover, the actual stability of the latent classes over time has yet to be established. On the other hand, our long-term experience with patients with AERD seems to suggest that such subphenotypes might well exist.

In conclusion, the application of a novel biostatical methodology allowed us to identify, for the first time, unique AERD subphenotypes that can occur in regular clinical practice. The encouraging results of the present study supply a body of evidence for the heterogeneity of the AERD population. This might prove quite helpful in any future studies on AERD, especially with regard to a better appreciation of this disorder and effective identification of patients exposed to a greater risk of adverse outcomes who require more individualized treatment.

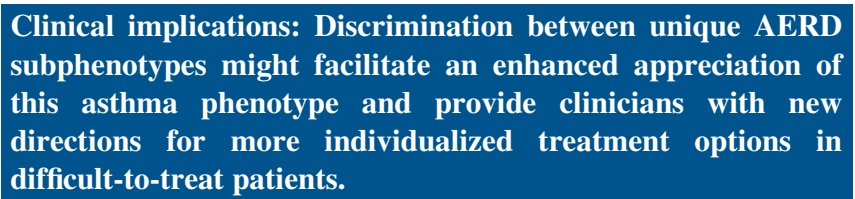

\section{REFERENCES}

1. Samter M, Beers R Jr. Intolerance to aspirin: clinical studies and consideration of its pathogenesis. Ann Intern Med 1968;68:975-83. 
2. Szczeklik A, Nizankowska E, Duplaga M. Natural history of aspirin-induced asthma. Eur Respir J 2000;16:432-6.

3. Stevenson DD, Szczeklik A. Clinical and pathologic perspectives on aspirin sensitivity and asthma. J Allergy Clin Immunol 2006;118:773-86.

4. Berges-Gimeno MP, Simon RA, Stevenson DD. The natural history and clinical characteristics of aspirin-exacerbated respiratory disease. Ann Allergy Asthma Immunol 2002;89:474-8.

5. Bochenek G, Nizankowska E, Szczeklik A. Atopy trait in hypersensitivity to nonsteroidal anti-inflammatory drugs. Allergy 1996;51:16-23.

6. Sanak M, Bochenek G, Faber L, Plutecka H, Szczeklik A. Elevated urinary leukotriene $\mathrm{E}_{4}$ excretion in asthma: a comparison of HPLC-mass spectrometry and ELISA. Allergy 2010;65:662-9.

7. Wenzel SE. Asthma: defining of the persistent adult phenotypes. Lancet 2006;368: 804-13.

8. Mascia K, Haselkorn T, Deniz YM, Miller DP, Bleecker ER, Borish L, et al. Aspirin sensitivity and severity of asthma: evidence for irreversible airway obstruction in patients with severe or difficult-to treat asthma. J Allergy Clin Immunol 2005;116:970-5.

9. Henderson J, Granell R, Heron J, Sheriff A, Simpson A, Woodcock A, et al. Association of wheezing phenotypes In the first six years of life with atopy, lung function and airway responsiveness. Thorax 2008;63:974-80.

10. Spycher BD, Silverman M, Brooke AM, Minder CE, Kuehni CE. Distinguishing phenotypes of childhood wheeze and cough using latent class analysis. Eur Respir J 2008;31:974-81.

11. Siroux V, Basagna X, Boudier A, Pin I, Garcia-Aymerich J, Vesin A, et al Identifying adult asthma phenotypes using a clustering approach. Eur Respir J 2011;38:310-7

12. Haldar P, Pavord ID, Dominic ES, Berry MA, Thomas M, Brightling CE, et al Cluster analysis and clinical asthma phenotypes. Am J Respir Crit Care Med 2008; 178:218-24.

13. Moore WC, Meyers DA, Wenzel SE, Teague WG, Li H, Li X, et al. Identification of asthma phenotypes using cluster analysis in the Severe Asthma Research Program. Am J Respir Crit Care Med 2009;181:315-23.

14. Fitzpatrick AM, Teague WG, Meyers DA, Peters SP, Li X, Li H, et al. Heterogeneity of severe asthma in childhood: confirmation by cluster analysis of children in the National Institutes of Health/National Heart, Lung, and Blood Institute Severe Asthma Research Program. Am J Respir Crit Care Med 2011; 127:382-9.

15. Nizankowska-Mogilnicka E, Bochenek G, Mastalerz L, Swierczyńska M, Picado $\mathrm{C}$, Scadding $\mathrm{G}$, et al. EAACI/GA2LEN guideline: aspirin provocation tests for diagnosis of aspirin hypersensitivity. Allergy 2007;62:1111-8.

16. National Heart, Lung, and Blood Institute, National Asthma Education and Prevention Program. Expert panel report 3: guidelines for the diagnosis and management of asthma. Bethesda: National Heart, Lung, and Blood Institute; 2007.

17. Hagenars JA, McCutheon AL. Applied latent class analysis. Cambridge (United Kingdom): Cambridge University Press; 2002.

18. Lanza ST, Collins LM, Lemmon DR, Schafer JL. PROC LCA: SAS procedure for latent class analysis. Struct Equ Modelling 2007;14:671-94.
19. Chhabra SK, Chhabra P. Gender differences in perception of dyspnoea, assessment of control, and quality of life in asthma. J Asthma 2011;48:609-15.

20. Rowe BH, Voaklander DC, Wang D, Senthilselvan A, Klassen TP, Marrie TJ, et al. Asthma presentation by adults to emergency departments in Alberta, Canada: a large population-based study. Chest 2009;135:57-65.

21. The ENFUMOSA Study Group. The ENFUMOSA cross-sectional European multicentre study of the clinical phenotype of chronic severe asthma. Eur Respir J 2003;22:470-7.

22. Kowalski ML, Cieslak M, Perez-Novo CA, Makowska JS, Bachert C. Clinical and immunological determinants of severe/refractory asthma (SRA): association with Staphylococcal superantigen-specific IgE antibodies. Allergy 2011;66:32-8.

23. Yoshimine F, Hasegawa T, Suzuki E, Terada M, Koya T, Kondoh A, et al. Contribution of aspirin-intolerant asthma to near fatal asthma based on a questionnaire survey in Niigata Prefecture, Japan. Respirology 2005;10:477-84.

24. Fukutomi Y, Taniguschi M, Tsuburai T, Tanimoto H, Oshikata C, Ono E, et al. Obesity and aspirin intolerance are risk factors for difficult-to treat asthma in Japanese non-atopic women. Clin Exp Allergy 2012;45:738-46.

25. Wearthall M, Travers J, Shirtcliffe PM, Marsh SE, Williams MV, Novitz MR, et al. Distinct clinical phenotypes of airways disease defined by cluster analysis. Eur Respir J 2009;34:812-8.

26. Mikos M, Grzanka P, Sladek K, Pulka G, Bochenek G, Soja J, et al. High-resolution computed tomography evaluation of peripheral airways in asthma patients: comparison of focal and diffuse air-trapping. Respiration 2009;77:381-8.

27. Mascia K, Borish L, Patrie J, Hunt J, Phillips CD, Steinke JW. Chronic hyperplastic eosinophilic sinusitis as a predictor of aspirin-exacerbated respiratory disease. Ann Allergy Asthma Immunol 2005;94:652-7.

28. Ponte EV, Franco R, Nascimento HF, Souza-Machado A, Cunha S, Baretto ML, et al. Lack of control of severe asthma is associated with co-existence of moderate-to-severe rhinitis. Allergy 2008;63:564-9.

29. Williamson PA, Vaidyanathan S, Clearie K, Barnes M, Lipworth BJ. Airway dysfunction in nasal polyposis: a spectrum of asthmatic disease? Clin Exp Allergy 2011;41:1379-85.

30. Fokkens WJ, Lund VJ, Mullol J, Bachert C, Alobid I, Baroody F, et al. EPOS 2012: European position paper on rhinosinusitis and nasal polyps 2012. A summary for otorhinolaryngologists. Rhinology 2012;50:1-12.

31. Hu Y, Cao PP, Liang GT, Cui YH, Liu Z. Diagnostic significance of blood eosinophil count in eosinophilic rhinosinusitis with nasal polyps in Chinese adults. Laryngoscope 2012;122:498-503.

32. Bochenek G, Nagraba K, Nizankowska E, Szczeklik A. A controlled study of $9 \alpha, 11 \beta-\mathrm{PGF}_{2}$ (a prostaglandin $\mathrm{D}_{2}$ metabolite) in plasma and urine of patients with bronchial asthma and healthy controls after aspirin challenge. J Allergy Clin Immunol 2003;111:743-9.

33. Higashi N, Tanigushi M, Haruhisa M, Kawagishi Y, Ishii T, Higashi A, et al. Clinical features of asthmatic patients with increased urinary leukotriene $\mathrm{E}_{4}$ excretion (hyperleukotrienuria): involvement of chronic hyperplastic rhinosinusitis with nasal polyposis. J Allergy Clin Immunol 2004;113:277-83.

34. Szefler S, Wenzel S, Brown R, Erzurum SC, Fahy JV, Hamilton RG, et al. Asthma outcomes: Biomarkers. J Allergy Clin Immunol 2012;129(suppl):S9-23. 


\section{METHODS}

Patients' data were collected by using a specifically structured questionnaire. Participants underwent a demographic and detailed medical history interview, including questions regarding current symptoms and clinical course of asthma, with special attention being directed to the age at onset. Patients were asked about the number of ED visits and hospitalizations in hospital wards and in the ICU because of asthma exacerbations throughout their asthma duration. The place where an exacerbation was treated reflected its severity. Current asthma treatment with regard to doses of oral and inhaled corticosteroids was carefully evaluated. Patients were also asked about current (during the last 3 months) and previous upper airway symptoms (nasal congestion, rhinorrhea, and loss of smell), occurrence of nasal polyps (currently or in the past), and number of polypectomies. In this way chronic rhinosinusitis was estimated. Medical documentation was reviewed for this purpose when feasible. Sinus computed tomographic scans were not performed for all patients: their results were estimated only for those patients who delivered such documentation.

\section{Asthma severity and control}

The assessment of asthma severity was based on the NAEPP EPR $3 .{ }^{\text {E1 Current }}$ asthma symptoms, use of bronchodilators, limitation of normal activity, current lung function, and frequency of exacerbations in the previous year were taken into account. Asthma was classified as follows: (1) intermittent, (2) mild persistent, (3) moderate persistent, and (4) severe persistent. An individual patient was allocated to the highest level in which any of those features occurred.

Asthma control was assessed through the Asthma Control Test. ${ }^{\mathrm{E} 2}$ This test consisted of 5 rating categories (1- to 5-point scale), which reflected the level of asthma control over the previous 4 weeks. The maximal score was 25 . Asthma was then categorized as follows: well controlled (score, 25), partially controlled (score, 20-24), or poorly controlled (score, <20).

\section{Spirometry}

Standard spirometry was carried out with an electronic spirometer (Master Screen; Jaeger, Wurzburg, Germany) at baseline and 15 minutes after administration of 4 puffs of salbutamol $(400 \mu \mathrm{g})$ by using a metered-dose inhaler Patients were asked to withhold the use of short-acting $\beta_{2}$-agonists for 6 hours and long-acting $\beta_{2}$-agonists for 12 hours before the measurements were to be taken. The best of 3 repeatable forced expiratory maneuvers was then recorded. The percent predicted values of $\mathrm{FEV}_{1}, \mathrm{FEV}_{1}$ /forced vital capacity ratio, and reversibility of $\mathrm{FEV}_{1}$ after bronchodilator were automatically calculated.

\section{Skin prick tests}

Skin prick tests with 12 common aeroallergens (Allergopharma, Reinbek, Germany) were carried out. Patients were tested with 12 standard aeroallergens (Dermatophagoides pteronyssinus, Dermatophagoides farinae, mixed grasses, alder, hazel, birch, motherwort, ribwort plantain, cat, dog, Alternaria species, and Cladosporium species). The test response was regarded as positive if the mean wheal diameter was $3 \mathrm{~mm}$ or greater than that elicited by the negative control (allergen diluent). The positive control was $1 \mathrm{mg} / \mathrm{mL}$ histamine. Patients were deemed atopic when at least 1 positive test response was obtained.

\section{Total IgE}

Total IgE levels were measured by using the nephelometric method with the NLatex IgE mono assembly kits (Dade Behring, Newark, NJ).

\section{Blood eosinophilia}

Blood eosinophil counts were calculated in the Fuchs-Rosenthal chamber.

\section{Measurement of $\mathrm{LTE}_{4}$ concentrations in urine}

The $\mathrm{LTE}_{4}$ concentration in urine was measured in the unpurified samples by using ELISA (Cayman Chemicals, Ann Arbor, Mich) with correction for creatinine content, as described elsewhere. ${ }^{\mathrm{E} 3}$

\section{Rationale behind specific presentation of exacerbations applied in this study}

Asthma exacerbations were expressed by the number of ED visits, hospitalizations, and ICU stays. It was found that the number of ED visits and hospitalizations caused by asthma exacerbations was positively correlated with asthma duration. On the basis of the Spearman rank correlation coefficient, correlations of an $r$ value of 0.38 with a $P$ value of less than .001 and an $r$ value of 0.40 with a $P$ value of less than .001 were found for ED visits and hospitalizations, respectively. Thus to eliminate the influence of asthma duration on the number of exacerbations, the numbers of ED visits and hospitalizations for each patient were not expressed in absolute values; instead, they were calculated per year of asthma duration. However, it was found that this approach yielded very low fractional numbers. Therefore the numbers of ED visits and hospitalizations were calculated per 10 years of asthma duration. Finally, the obtained results expressing the frequency of ED visits/hospitalizations (calculated per 10 years of asthma duration) were divided into the following 3 intervals (tertiles): first tertile, low frequency; second tertile, moderate frequency; and third tertile, high frequency. Thus the choice of 10 years was arbitrary and based on the fact that the asthma duration ranged from 10 months to 52 years (median, 19 years), and the numbers of patients with asthma duration in 10 -year periods of time were distributed almost evenly: 10 years or less, $47(23.4 \%)$ patients; 11 to 20 years, $65(32.3 \%)$ patients; 21 to 30 years; 58 (28.9\%) patients; and 30 years or more, $31(15.4 \%)$ patients. Furthermore, this approach was statistically useful because it made it possible to categorize the variables.

Because hospitalizations in the ICU caused by asthma exacerbations were much less frequent and affected only some patients, the subjects were only categorized into those who were or were not hospitalized in the ICU throughout their asthma duration.

\section{Analytic strategy}

Latent class models were fitted to the clinical, functional, and laboratory variables measured in the study. ${ }^{\mathrm{E}, \mathrm{E} 5}$ This statistical modeling technique was used to estimate the number of classes of an underlying categorical latent variables with a finite number of mutually exclusive levels, which simultaneously considered the relationships between the respective numbers of observed variables. The underlying principle for the model is that the variables under consideration are the imperfect indicators of the unobserved (latent) variables; accordingly, "latent" refers to not being visible directly but identified through a set of manifest indicators. Therefore LCA is particularly appropriate for modeling the data on the occurrence or absence of disease symptoms. When latent classes represent the manifestation of a disease, they can be regarded as bona fide disease phenotypes.

In a latent class model 2 types of parameters were estimated: the prevalence of each latent class (ie, the a priori probability that a randomly selected subject was in each class) and the conditional probabilities describing the distribution of the responses to each question within each class. Identification of the optimal model was done in a stepwise manner. In this analysis sequential models with the numbers of classes starting from 1 to 5 were fitted and compared (Table E1). The final choice of a latent class model was based on the likelihood ratio $\chi^{2}$ statistic $\mathrm{G}^{2}$ and, on the other hand, the Akaike information criterion and the sample size-adjusted Bayesian information criterion.

The lowest values of these criteria indicated the superior model (ie, the model that best balanced the number of parameters and the information yielded by those parameters). When the best model was determined, each subject was then allocated to a single latent class based on the maximum probability assignment rule (ie, to the class with the highest a posteriori probability of membership). Table E2 reveals the a posteriori class assignment probability for the 4-class solution. High diagonal values, compared with the low off-diagonal values, indicate that the adopted classification approach seems appropriate (Table E2).

\section{Selection of variables}

The assumption of LCA is that variables are categorical and not continuous and that they are independent of one another. To comply with this condition, 
we searched the available dataset and selected the following independent categorical variables relevant to the study:

(1) age at asthma onset (childhood onset, $<12$ years; adult onset, $\geq 12$ years);

(2) body mass index (nonobese, $<30 \mathrm{~kg} / \mathrm{m}^{2}$; obese, $\geq 30 \mathrm{~kg} / \mathrm{m}^{2}$ );

(3) current level of asthma control according to the Asthma Control Test (1, well controlled; 2, partially controlled; and 3, uncontrolled);

(4) frequency of ED visits caused by asthma exacerbations calculated per 10 years of entire asthma duration (first tertile, low frequency: $<0.63$ visits per 10 years of asthma duration; second tertile, moderate frequency: $0.63-2.57$ visits per 10 years of asthma duration; and third tertile, high frequency: $\geq 2.57$ visits per 10 years of asthma duration);

(5) frequency of hospitalizations caused by asthma exacerbations calculated per 10 years of entire asthma duration (first tertile, low frequency: $<0.5$ hospitalizations per 10 years of asthma duration; second tertile, moderate frequency: $0.5-2.11$ hospitalizations per 10 years of asthma duration; and third tertile, high frequency: $\geq 2.11$ hospitalizations per 10 years of asthma duration);

(6) hospitalizations in the ICU caused by asthma exacerbations (yes; no);

(7) upper airway symptoms during the 3 months before the study (ie, nasal congestion, rhinorrhea, or loss of smell [yes; no]);

(8) occurrence of nasal and sinus polyps at the time of the study or in the past (yes; no);

(9) polypectomies or sinus surgeries throughout the duration of the disease (yes; no);

(10) $\mathrm{FEV}_{1}$ percent predicted (bronchial obstruction, $<80 \%$; normal lung function, $\geq 80 \%$ );

(11) $\triangle \mathrm{FEV}_{1}$ after bronchodilator (not reversible obstruction, $<12 \%$; reversible obstruction, $\geq 12 \%$ );

(12) positive skin prick test response to at least 1 aeroallergen (yes; no)

(13) total IgE level ( $\geq 100 \mathrm{IU} / \mathrm{mL} ;<100 \mathrm{IU} / \mathrm{mL})$; and

(14) blood eosinophil count ( $\geq 400 / \mathrm{mL} ;<400 \mathrm{~mL}$ ).

The effects of sex and asthma duration were additionally estimated as covariates included in the final 4-class model. The class membership probabilities were estimated separately for male and female subjects by using sex as a categorical covariate. Asthma duration was estimated as a covariate because of its continuity and to preclude its effect on the number of exacerbations.

After identifying the 4 final classes, the mean values of the logarithmically transformed urinary $\mathrm{LTE}_{4}$ concentrations were calculated and compared among the respective classes. The level of urinary $\mathrm{LTE}_{4}$ was not included in the LCA because it was a continuous variable, and the cutoff point between normal and increased levels has not yet been estimated.

In the following stage the treatment of asthma in each latent class was assessed. The present treatment of asthma as a variable was not included in the LCA because it could affect some categorical variables involved in the analysis and might also consequently modify the course of asthma. Treatment of asthma with corticosteroids was divided into 4 categories: 1 , no corticosteroids; 2, inhaled corticosteroids in a daily dose of $500 \mu \mathrm{g}$ or less of fluticasone equivalent; 3 , inhaled corticosteroids in a daily dose of greater than $500 \mu \mathrm{g}$ of fluticasone equivalent; and 4 , oral corticosteroids together with inhaled corticosteroids. In each class the proportion of patients using each category of treatment was computed.

Finally, the entire group was assessed with respect to the current level of asthma severity according to the NAEPP EPR 3 criteria: 1 , intermittent; 2 , mild persistent; 3 , moderate persistent; and 4 , severe persistent. In each class the proportion of patients representing each level of asthma severity was determined.

\section{RESULTS}

On the basis of the lowest Akaike information criterion, the Bayesian information criterion, and an entropy value of 0.82 , the best 4-class model was determined through the application of LCA. Parameter estimates for this model are comprised in Table E3.

Logistic regression analysis revealed that the odds for the allocation to a particular class was related to the duration of asthma (Table E4).

\section{REFERENCES}

E1. National Heart, Lung, and Blood Institute, National Asthma Education and Prevention Program. Expert panel report 3: guidelines for the diagnosis and management of asthma. Bethesda: National Heart, Lung, and Blood Institute; 2007.

E2. Nathan RA, Sorkness CA, Kosinski M, Schatz M, Li JT, Marcus P, et al. Development of the asthma control test: a survey for assessing asthma control. J Allergy Clin Immunol 2004;113:59-65.

E3. Kumlin M. Measurement of leukotrienes in humans. Am J Respir Crit Care Med 2000;161:102-6.

E4. Hagenars JA, McCutheon AL. Applied latent class analysis. Cambridge (United Kingdom): Cambridge University Press; 2002.

E5. Lanza ST, Collins LM, Lemmon DR, Schafer JL. PROC LCA: SAS procedure for latent class analysis. Struct Equ Modelling 2007;14:671-94. 
TABLE E1. Measure of fitting for the latent classes

\begin{tabular}{lcccc}
\hline No. of classes & LL & AIC & BIC & Entropy \\
\hline 1 & -1844.33 & 1604.58 & 1606.88 & 1.0 \\
2 & -1788.01 & 1527.95 & 1532.68 & 0.68 \\
3 & -1764.67 & 1517.28 & 1692.36 & 0.85 \\
4 & -1741.53 & $\mathbf{1 5 0 7 . 0 0}$ & $\mathbf{1 5 1 6 . 6 0}$ & $\mathbf{0 . 8 2}$ \\
5 & -1723.72 & 1507.38 & 1519.41 & 0.84 \\
\hline
\end{tabular}

The boldface values represent that the 4-class model was the best fit to the data.

$A I C$, Akaike information criterion; BIC, Bayesian information criterion (adjusted to sample size); $L L, \log$ likelihood. 
TABLE E2. Assignment probability by class

\begin{tabular}{lcccc}
\hline & Class 1 & Class 2 & Class 3 & Class 4 \\
\hline Class 1 & $\mathbf{0 . 8 1 8}$ & 0.152 & 0.027 & 0.004 \\
Class 2 & 0.042 & $\mathbf{0 . 9 1 9}$ & 0.024 & 0.015 \\
Class 3 & 0.043 & 0.051 & $\mathbf{0 . 8 8 6}$ & 0.019 \\
Class 4 & 0.000 & 0.001 & 0.002 & $\mathbf{0 . 9 9 6}$ \\
\hline
\end{tabular}

High diagonal (boldface) values indicate that the adopted classification approach was appropriate. 
TABLE E3. Estimates with SEs for the 4-class model determined by using LCA

\begin{tabular}{|c|c|c|c|c|}
\hline & Class 1 & Class 2 & Class 3 & Class 4 \\
\hline Male subjects & $0.13(0.05)$ & $0.47(0.06)$ & $0.40(0.05)$ & $0.00(0.00)$ \\
\hline Female subjects & $0.20(0.04)$ & $0.33(0.05)$ & $0.37(0.04)$ & $0.10(0.03)$ \\
\hline \multicolumn{5}{|l|}{ Age at the onset of asthma } \\
\hline$<12 \mathrm{y}$ & $0.08(0.05)$ & $0.00(0.00)$ & $0.00(0.00)$ & $0.46(0.18)$ \\
\hline \multicolumn{5}{|l|}{ BMI } \\
\hline$\geq 30 \mathrm{~kg} / \mathrm{m}^{2}$ & $0.05(0.05)$ & $0.17(0.05)$ & $0.23(0.05)$ & $0.41(0.16)$ \\
\hline 1: Well controlled & $0.20(0.08)$ & $0.36(0.06)$ & $0.05(0.03)$ & $0.17(0.12)$ \\
\hline 2: Partially controlled & $0.51(0.10)$ & $0.40(0.07)$ & $0.24(0.06)$ & $0.12(0.12)$ \\
\hline 3: Uncontrolled & $0.29(0.10)$ & $0.24(0.06)$ & $0.71(0.06)$ & $0.71(0.15)$ \\
\hline \multicolumn{5}{|l|}{ ED visits* } \\
\hline First tertile $(<0.63$ per $10 \mathrm{y})$ & $0.33(0.10)$ & $0.48(0.07)$ & $0.25(0.06)$ & $0.01(0.03)$ \\
\hline Second tertile $(0.63-2.57$ per $10 \mathrm{y})$ & $0.36(0.10)$ & $0.38(0.07)$ & $0.31(0.06)$ & $0.15(0.13)$ \\
\hline Third tertile $(>2.11$ per $10 \mathrm{y})$ & $0.48(0.11)$ & $0.08(0.05)$ & $0.44(0.07)$ & $0.67(0.16)$ \\
\hline \multicolumn{5}{|l|}{ Hospitalizations in ICU } \\
\hline Yes & $0.03(0.04)$ & $0.10(0.04)$ & $0.37(0.06)$ & $0.80(0.13)$ \\
\hline No & $0.97(0.04)$ & $0.90(0.04)$ & $0.63(0.06)$ & $0.20(0.13)$ \\
\hline \multicolumn{5}{|l|}{ Upper airway symptoms } \\
\hline Yes & $1.00(0.01)$ & $0.70(0.06)$ & $0.85(0.05)$ & $0.68(0.15)$ \\
\hline No & $0.00(0.01)$ & $0.30(0.62)$ & $0.15(0.05)$ & $0.32(0.15)$ \\
\hline \multicolumn{5}{|l|}{ Nasal polyps whenever } \\
\hline Yes & $0.96(0.07)$ & $0.80(0.06)$ & $0.78(0.05)$ & $0.55(0.17)$ \\
\hline No & $0.04(0.07)$ & $0.20(0.06)$ & $0.22(0.05)$ & $0.45(0.17)$ \\
\hline \multicolumn{5}{|l|}{ Polypectomies whenever } \\
\hline Yes & $0.81(0.08)$ & $0.94(0.05)$ & $0.72(0.06)$ & $0.66(0.21)$ \\
\hline Yes & $0.59(0.11)$ & $0.52(0.07)$ & $0.47(0.06)$ & $0.63(0.15)$ \\
\hline No & $0.41(0.11)$ & $0.48(0.07)$ & $0.53(0.06)$ & $0.37(0.15)$ \\
\hline \multicolumn{5}{|l|}{ IgE total } \\
\hline$\geq 100 \mathrm{IU} / \mathrm{mL}$ & $0.67(0.12)$ & $0.16(0.06)$ & $0.60(0.06)$ & $0.45(0.16)$ \\
\hline$<100 \mathrm{IU} / \mathrm{mL}$ & $0.37(0.12)$ & $0.84(0.06)$ & $0.40(0.06)$ & $0.55(0.16)$ \\
\hline \multicolumn{5}{|l|}{ Blood eosinophil counts } \\
\hline$\geq 400 / \mathrm{mL}$ & $0.63(0.12)$ & $0.31(0.06)$ & $0.40(0.06)$ & $0.41(0.16)$ \\
\hline$<400 / \mathrm{mL}$ & $0.37(0.12)$ & $0.69(0.06)$ & $0.60(0.06)$ & $0.59(0.16)$ \\
\hline
\end{tabular}

$A C T$, Asthma Control Test; BMI, body mass index.

*Throughout the time of asthma duration. 
TABLE E4. Odds for allocation to latent classes relative to the reference class corresponding to a 1-year increase in duration of asthma

Class 1

Male subjects

Female subjects

$1.09(0.19-6.07)$

$0.90(0.84-0.95)$

Values are presented as odds ratios $(95 \% \mathrm{CIs})$.
Class 2

$2.84(1.06-7.6)$

$0.97(0.93-1.01)$

Class 3

Class 4

1.0

1.0

$1.07(1.01-1.14)$ 